\title{
On the Saturation Effects and Start Jump of Gaussian Modes in Oscillators
}

\author{
W. J. WITTEMAN AND G. J. ERNST
}

\begin{abstract}
The present paper deals with the interaction of a Gaussian mode with a homogeneous and an inhomogeneous laser transition. The interaction of the beam with the medium in a laser is treated by taking into account the spatial distribution of both radiation and gain. The intensity characteristics are very different from those obtained for a one-dimensional interaction of a plane wave with a saturating medium. In the presence of a small-signal gain profile the threshold condition requires much higher inversion densities along the optic axis than by ignoring this profile. For gas lasers, for instance, having a small-signal gain profile that is approximately described by a zero-order Bessel function, the threshold inversion density can be about 50 percent higher. For high-power systems the saturation of the medium by the Gaussian intensity distribution results in a considerable amount of radial radiation transport. For homogeneous transitions this amount is about equal to the stimulated emission and for inhomogeneous transitions it is about haif of it, independent of the beamwidth. Further, it is found that if one slowly passes the threshold condition for laser action, the intensity jumps from zero to a certain value and vice versa. This effect has also been verified experimentally.
\end{abstract}

\section{INTRODUCTION}

$\mathrm{T}$ HE radiation intensities produced by steady-state lasers have been computed in the past by using one-dimensional interaction of a plane wave with a saturating medium [1], [2] . As far as the interaction is concerned, the resonator has been assumed to support plane electromagnetic waves with its outcoupling loss concentrated near the mirrors. By using this approximation, one calculates the intensity characteristics, maximum output flux densities, etc., as a function of gain and outcoupling loss for lines with homogeneous and inhomogeneous broadening. However, in any interaction phenomenon of an electromagnetic beam and an amplifying medium there is no plane wave and no uniform gain of the field due to the finite aperture, to the inhomogeneity of the medium, and/or to the intensity-induced saturation of the medium. This nonuniformity depends not only on the coordinate of the direction of propagation, but also on the coordinates perpendicular to the direction of propagation, i.e., the gain profile.

In search of maximum radiation production from an efficiently pumped optical system, it seems advisable to extend the intensity computations by removing the restrictions of only one-dimensional interaction. Usually, the spatial distribution of the radiation intensity is also computed without taking into account a nonuniform gain profile. But this turns out to be very reasonable, because as far as the radiation distribution is concerned it is found [3] that, at least for small

Manuscript received September 21, 1974; revised January 15, 1975.

The authors are with the Department of Physics, Twente University of Technology, Enschede, The Netherlands. laser systems, the geometry of the beam is practically unaltered by the inhomogeneity of the gain, so that the optical beam parameters such as beamwidth can be reasonably well predicted by neglecting the gain profile. This is found for a positive as well as a negative gain profile, because in both cases a change in amplitude distribution, which is expected by neglecting phase changes, is nearly canceled by the effect of phase changes. Therefore, it is found and in agreement with observations, that under normal experimental conditions of laser systems the effect of a gain profile, caused either by the smallsignal gain or by the saturation, will leave the beam size practically unchanged. Thus there is no focusing or defocusing by the gain profile. However, we cannot neglect the gain profile for radiation-intensity studies. Here the gain profile, as we shall see, is an important parameter, even for small systems having low output power.

The analysis here treats homogeneous and inhomogeneous laser transitions. We restrict ourselves to a commonly used system of a single-mode Gaussian beam in a radially symmetric excited medium. The advantage of treating this system is its simplicity. The physics involved can be easily appreciated and the effects of various processes on the radiation intensity can be compared. It is found that the three-dimensional character of the gain has for any optimized laser system-high or low power, homogeneous or inhomogeneous transitions-a large bearing on the intensity characteristics and output powers. Furthermore, from the computation of the intensity characteristics a remarkable effect can be predicted: i.e., when a laser builds up the inversion and passes (slowly) the threshold condition for the Gaussian mode, the laser output does not start from zero, but jumps to a finite value. We verified this jump experimentally, which gives a direct proof of the so-called "radial radiation transport." Although the analysis is primarily set up to describe saturation effects and to compute laser characteristics, it is also seen that the threshold conditions are changed drastically by a small-signal gain profile.

\section{The INCREMENTAL GAIN}

Since in the present analysis we are dealing with the propagation of a Gaussian beam in a normal resonator, the nonuniformity of the medium must be lenslike in order to preserve the Gaussian nature. This means that the medium is described by a quadratic profile. In fact, the appearance of high-power Gaussian laser beams supports the assumption that a quadratic description can be adequate. In practice, of course, no profile is quadratic, but it should be noted that for media having axial symmetry a series expansion of the propagation constant in terms of the radial distance from the axis 
shows that the main terms near the axis (where the field is strongest) are the constant and quadratic ones.

The derivation [4] of the propagation laws for Gaussian beams in quite general lenslike media starts with the scalar wave equation

$$
\nabla E+k^{2} E=0
$$

where the quadratic dependence of $k^{2}$ near the optic axis is given by

$$
k^{2}(r, z)=k_{0}^{2}(z)+k_{0}(z) k_{2}(z) r^{2} .
$$

These medium constants are generally complex to allow for loss or gain. $k_{0}$ is the part of the propagation constant that does not depend on $r$, whereas $k_{2}$ describes the variation of the propagation constant with $r$ and includes thermal effects, gain variations, and also the associated dispersive effects of the gain profile.

In solving (1), the main interest is in waves bouncing back and forth between two mirrors that propagate primarily in the $z$ direction, or almost-plane waves. It can be shown that such waves with a Gaussian radial dependence satisfy (1). Accordingly, one writes

$$
E(r, z)=E_{1} \exp \left\{-i\left(\phi+T+\frac{1}{2} r^{2} k_{0} / q\right)\right\}
$$

in which the time-dependent part has been dropped, $d \phi / d z=$ $k_{0}(z)$ is the complex propagation constant on the axis, $T$ is a complex phase parameter, $q$ is the complex beam parameter defined by

$$
\frac{1}{q}=\frac{1}{R(z)}-i \frac{\lambda}{\pi \omega^{2}(z)}
$$

where $R(z)$ is the radius of curvature of the wavefront, $\lambda$ is the wavelength, and $\omega(z)$ is the beam waist. Substitution of (3) into (1) gives, after comparison of terms of equal powers of $r$, the following two differential equations:

$$
\begin{aligned}
& T^{\prime}=-i\left(\frac{1}{q}+\frac{k_{0}^{\prime}}{2 k_{0}}\right) \\
& \frac{1}{q^{2}}+\left(\frac{1}{q}\right)^{\prime}+\frac{k_{0}^{\prime}}{k_{0}} \frac{1}{q}-\frac{k_{2}}{k_{0}}=0
\end{aligned}
$$

where the prime means differentiation with respect to $z$. For laser systems, (2) can be approximated by

$$
k=k_{0}+\frac{1}{2} k_{2} r^{2} \text {. }
$$

By separating real and imaginary parts, we write

$$
\begin{aligned}
& k_{0}=\beta_{0}+i \alpha_{0} \\
& k_{2}=\beta_{2}+i \alpha_{2} .
\end{aligned}
$$

By differentiating (3) with respect to $z$ and using (5), we find for $E$ on the axis ( $r=0)$

$$
\frac{1}{E} \frac{d E}{d z}=-i\left(k_{0}-\frac{i}{q}-\frac{i k_{0}^{\prime}}{2 k_{0}}\right)
$$

By adding the complex conjugates to both sides of (10), using
(4), and assuming that the wavelength is constant along the axis, the incremental gain along the axis is found to be

$$
\frac{1}{I} \frac{d I}{d z}=2\left(\alpha_{0}-\frac{1}{R(z)}\right)
$$

By substituting (4), (8), and (9) into (6) and taking the imaginary part, we get

$$
\frac{2}{R(z)}=\frac{d \ln \omega^{2}}{d z}-\frac{1}{2} \alpha_{2} \omega^{2} .
$$

By substituting (12) into (11), we finally get for the incremental gain on the optic axis

$$
\frac{1}{I} \frac{d I}{d z}=2 \alpha_{0}-\frac{d \ln \omega^{2}}{d z}+\frac{1}{2} \alpha_{2} \omega^{2} .
$$

The first term on the right-hand side of (13) describes the local conversion of inverted states into stimulating emission. This is the same term as found for a one-dimensional treatment with plane waves [5]. The last two terms on the righthand side of (13), equal to $2 / R(z)$, describe the part of the incremental gain due to convergence or divergence of the wavefront of a propagating wave [6]. One part of it, the second term of (13), is related to the continuity of propagating electromagnetic energy; in the absence of any interaction, the product of beam cross section and intensity at the optic axis of a Gaussian beam $\left(\omega^{2} I\right)$ is constant along the axis. Therefore this term simply describes focusing or defocusing of the beam. The last term on the right-hand side of (13) describes a contribution of the radial radiation transport to the incremental gain related to the parabolic gain profile. From (13) we obtain for the increase of energy flux

$$
\frac{d \ln \left(I \omega^{2}\right)}{d z}=2 \alpha_{0}+\frac{1}{2} \alpha \omega^{2} \text {. }
$$

It is seen that only the imaginary parts of the propagation constants, i.e., the gain and its profile, determine the increase of energy flux. Thermal and dispersive effects contribute in so far as they influence the beamwidth of the Gaussian mode [3]. But normally, the fractional change of beamwidth by thermal and dispersive effects is relatively small so that the incremental energy flux is practically given by the gain parameters.

In a laser system, the standing wave consists of a running wave in the $+z$ direction and one in the $-z$ direction. Although the beamwidths of the two running waves are not exactly equal, it turns out that in practice they can be taken equal at any place along the axis. If we write down (14) for both running waves, we then find because of bidirectional gain

$$
\frac{d \ln \left(I \omega^{2}\right)_{+}}{d z}=-\frac{d \ln \left(I \omega^{2}\right)_{-}}{d z}
$$

and we see that

$$
\left(I \omega^{2}\right)_{+}\left(I \omega^{2}\right)_{-}=\text {constant }
$$

Thus the product of the energy flux of the two running waves is constant along the axis. 


\section{Homogeneous Line Broadening}

For homogeneous line broadening, the saturated amplitude gain coefficient $\alpha$ is related to the unsaturated or small-signal gain coefficient $G$, which depends on the line shape of the transition by

$$
\alpha(r)=\frac{\frac{1}{2} G(r)}{1+I_{t}(r) / I_{0}}
$$

where $I_{0}$ is the saturation parameter and $I_{t}$ the total intensity of the two running waves. Since we are interested in the constant and quadratic term of the gain profile, we substitute into (17) for the unsaturated gain profile

$$
G(r)=G\left(1+\frac{1}{2} \gamma r^{2}\right)
$$

and for intensity

$$
I_{t}(r)=I_{t} \exp \left(-2 r^{2} / \omega^{2}\right)
$$

For the constant term of the amplitude gain we obtain

$$
\alpha_{0}=\frac{\frac{1}{2} G}{1+I_{t} / I_{0}} .
$$

For the quadratic term we get

$$
\alpha_{2}=\frac{\frac{1}{2} G}{1+I_{t} / I_{0}}\left(\gamma+\frac{4}{\omega^{2}} \frac{I_{t} / I_{0}}{1+I_{t} / I_{0}}\right)
$$

By substituting (20) and (21) into (14), we find for the incremental gain

$$
\frac{d \ln \left(I \omega^{2}\right)}{d z}=\frac{G}{1+I_{t} / I_{0}}\left(1+\frac{1}{4} \gamma \omega^{2}+\frac{I_{t} / I_{0}}{1+I_{t} / I_{0}}\right) .
$$

It is seen that the contribution of radial radiation transport to the incremental gain comes from both the small-signal gain profile and the gain induced saturation. In general, the smallsignal gain decreases with the distance from the axis ( $\gamma$ is negative). Such a profile broadens the wavefront and radiation energy is radially transported out of the center. This means that the threshold condition to start oscillations requires higher inversion densities along the axis than that predicted on the basis of one-dimensional plane-wave interaction.

This conclusion can also be reached more simply by stating that the integrated gain over the mode volume must be equal to the losses. Then one finds that the inversion along the axis is much higher than the average inversion needed to reach threshold. It is assumed, however, that the geometry of the beam is unchanged by the gain profile. In other words, it is assumed that if the effective diameter of the gain region has become smaller, the effective beam diameter does not become smaller. Only a more comprehensive analysis will show that the beamwidth is indeed practically unchanged by the gain profile [3].

In order to estimate the threshold condition, let us consider a small-signal gain profile proportional to the electron density of the discharge. In dealing with a positive column discharge, this gain profile can then be described by a zero-order Bessel function. The quadratic term of such a gain profile is

$$
\gamma=-\frac{2.88}{\rho^{2}}
$$

where $\rho$ is the radius of the tube diameter.

The second term of (22) becomes $-0.72 \omega^{2} / \rho^{2}$. In many single-mode oscillators the value of $\omega^{2} / \rho^{2}$ is about $\frac{1}{2}$. Substituting this value into (23) makes the incremental gain for starting oscillations about 36 percent smaller than in the case where the small-signal gain profile is ignored.

The last term on the right-hand side of (22) describes the part of the radial radiation transport that comes from the change of the saturated gain profile by the Gaussian intensity distribution. For optimized laser systems, when $I_{t} / I_{0} \gg 1$ the last term of (22) approaches one. Thus at high intensities the intensity profile itself leads to a radial radiation transport that contributes as much to the incremental gain as the stimulated emission from the local medium. It is interesting to note that the latter conclusion is independent of the beamwidth.

In order to find the intensity characteristics of an oscillator, we have to integrate (22) over a round trip through the resonator. In general, the beamwidth may vary considerably along the tube, so that the integration of (22) with the aid of (16) has to be worked out numerically. The integration becomes complicated because one also has to know the beamwidth at any place along the tube. The beamwidth in turn depends on $\alpha_{2}$ and, in fact, on $I_{t}$. However, the general behavior of the obtained characteristics is similar to what will be obtained for the case where $\lambda L / \pi \omega^{2}<<1$ ( $L$ is the length of the system), so that the beamwidth varies very little along the axis, and the outcoupling is not too large. In that case we find from (16) that the product of the intensities of the two running waves is constant. Let us consider a system having one totally reflecting mirror and one outcoupling mirror with reflectivity $R$. The maximum relative variation of the sum of the two intensities is equal to $\frac{1}{2} \sqrt{R}(1+1 / R)$. It is seen that even for $R=50$ percent the sum of the two intensities is constant to within 5 percent.

By integrating (22) over a round trip through the resonator and taking $I_{t}$ as constant, we find

$$
-\frac{\ln R}{2 G L}=\frac{1}{1+I_{t} / I_{0}}\left(1+0.25 \gamma \omega^{2}+\frac{I_{t} / I_{0}}{1+I_{t} / I_{0}}\right) .
$$

The outcoupled intensity $I_{\text {out }}$ is related to the total intensity within the laser by

$$
I_{\text {out }}=\frac{1-R}{1+R} I_{t}
$$

Normalized values of the output intensity are plotted in Fig. 1 as a function of $-G L / \ln R$ for various values of $\epsilon=1+$ $0.25 \gamma \omega^{2}$. In Fig. 2 we plotted the normalized output as a function of the reflectivity $R$ for various values of $G L$ and $\epsilon$. It is seen that the threshold condition depends on the smallsignal gain profile given by $\epsilon$.

Furthermore, it can be seen that there are nonzero fields with smaller gain over loss ratios than at threshold. These 


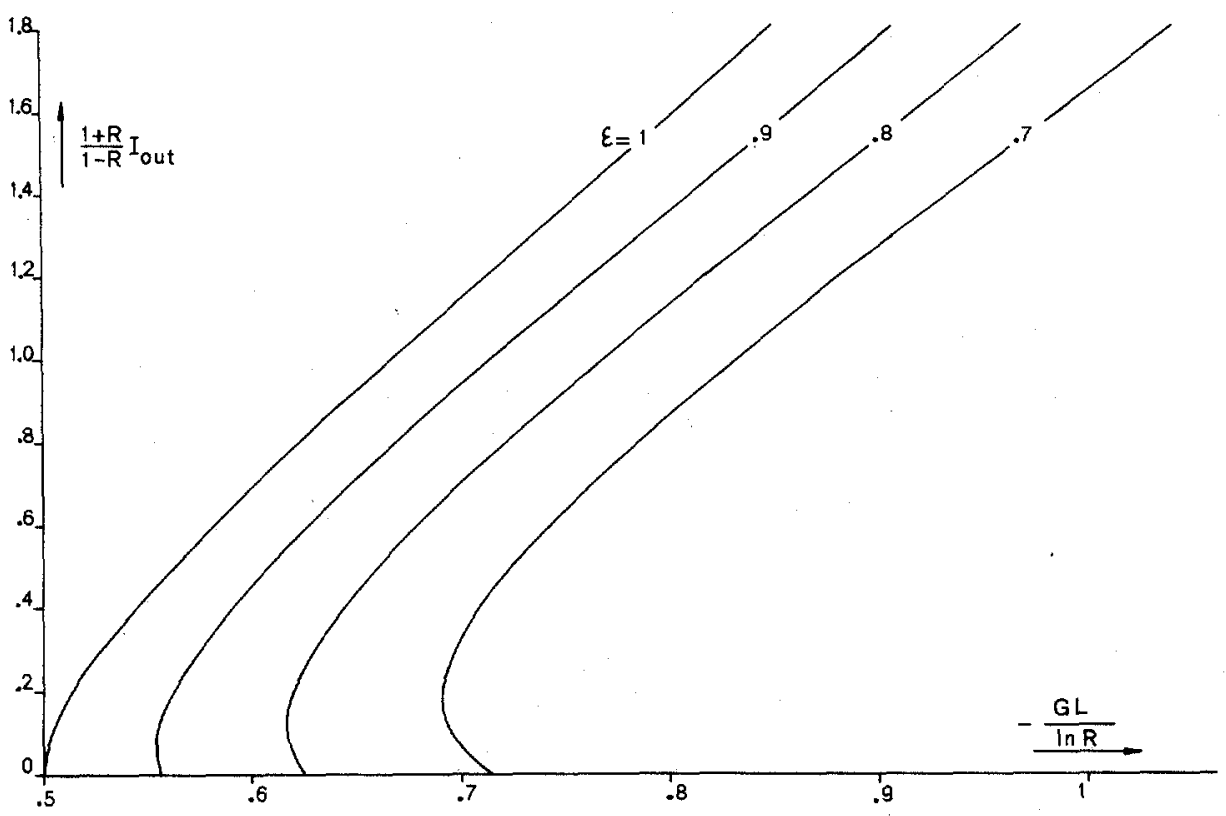

Fig. 1. The outcoupled intensity of homogeneous line broadening divided by the saturation-intensity parameter $I_{S}$ is plotted versus the gain over loss ratio. The small-signal gain profile is indicated by $\epsilon=1+0.25 \gamma \omega^{2}$.

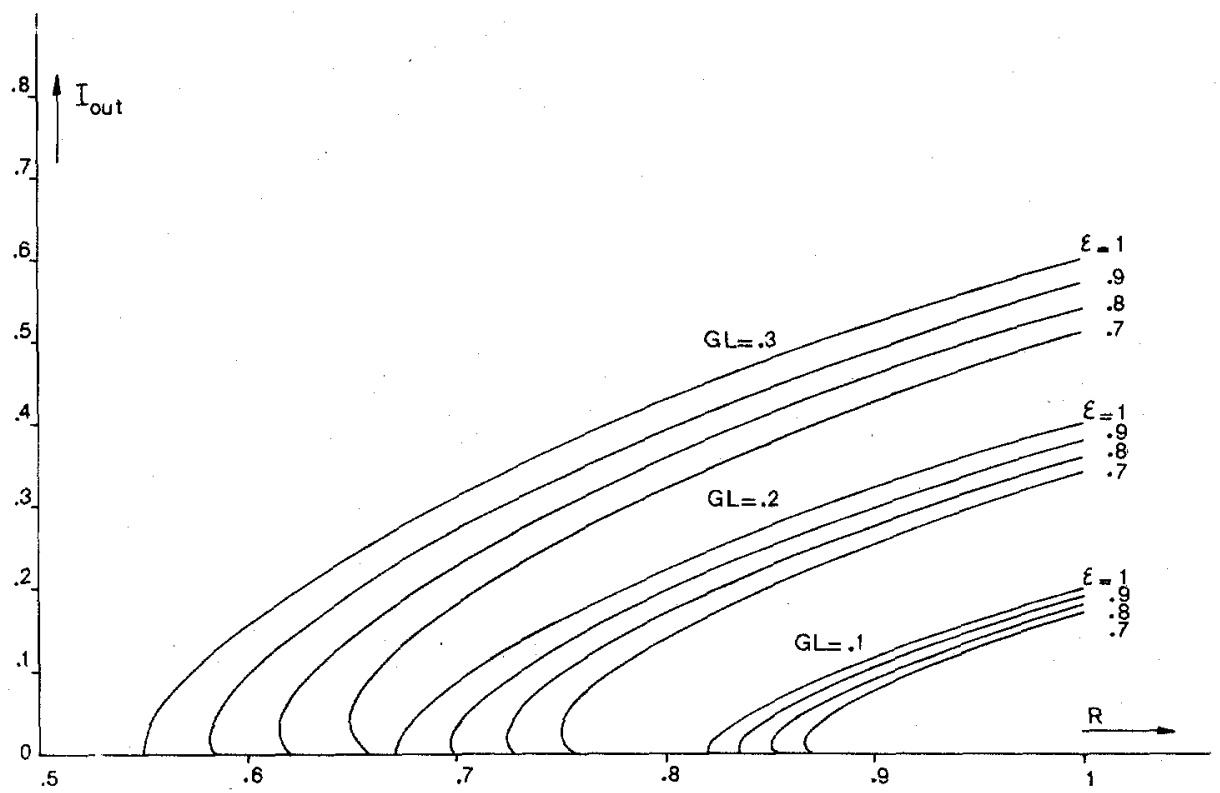

Fig. 2. The outcoupled intensity divided by $I_{S}$ is plotted as a function of $R$ for homogeneous line broadening. The characteristics indicate that near threshold the intensity is not continuously changeable, but jumps.

nonzero-field conditions can only be reached by a field that has already been built up within the resonator and therefore one first has to pass the zero-field threshold condition. By passing this point, the induced saturation levels the gain profile and, consequently, the divergence of the wavefront decreases, which means that less radiation is transported radially out of the center. Thus the apparent "loss factor" induced by the small-signal gain profile is reduced by the field itself and there- fore, if for a Gaussian beam system the gain over loss ratio is slowly increased from below threshold, the intensity does not increase continuously from zero as one passes threshold but jumps to a certain value.

The jump depends on the small-signal gain profile. The stronger this profile, i.e., the smaller $\gamma$, the larger the start jump. For values of $\gamma$ larger than or equal to zero there is no jump. 


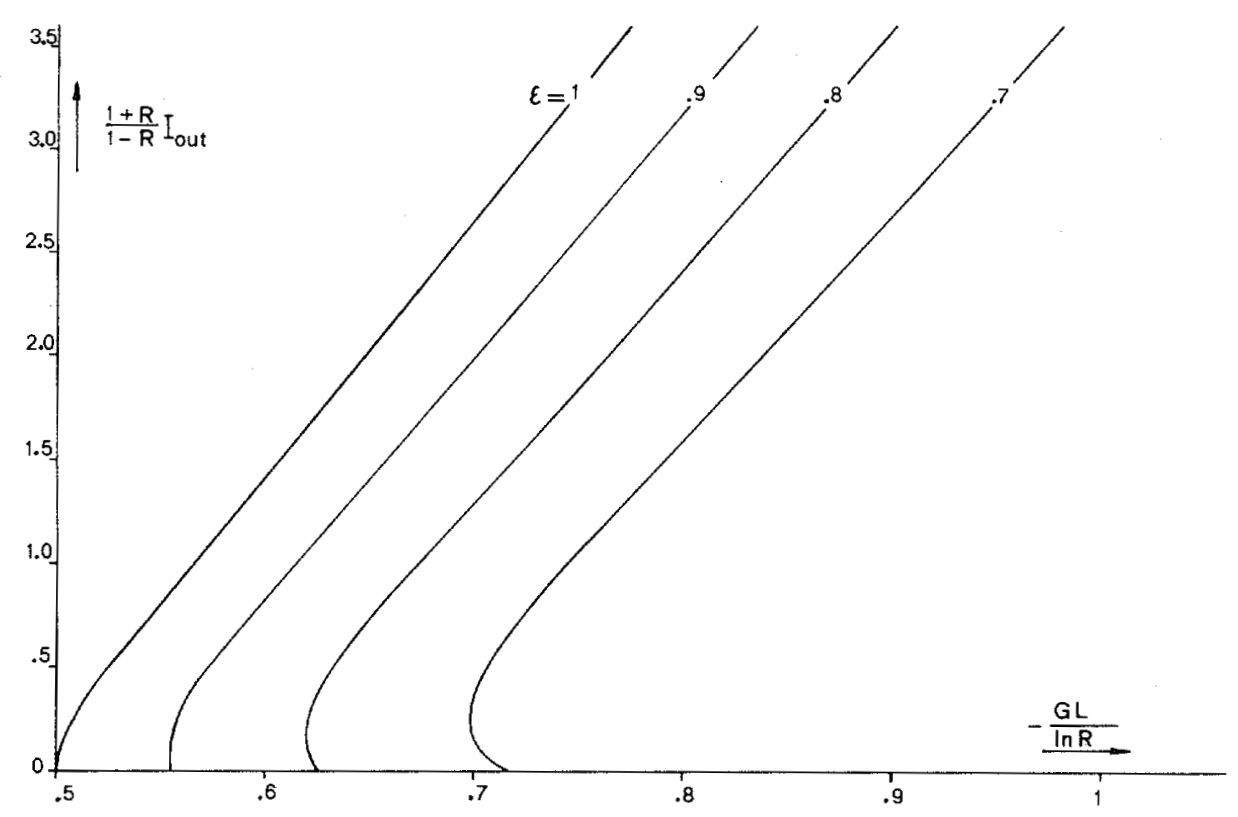

Fig. 3. The outcoupled intensity for inhomogeneous line broadening divided by the saturation parameter $I_{s}$ is plotted versus the gain over loss ratio.

\section{INHOMOGENEOUS LINE BROADENING}

For inhomogeneous line broadening the saturated amplitude gain coefficient is known as

$$
\alpha(r)=\frac{\frac{1}{2} G(r)}{\left\{1+I_{t}(r) / I_{0}\right\}^{1 / 2}}
$$

where $G(r)$ is again the small-signal intensity gain which depends on the line shape of the oscillating transition. Let us consider again that the small-signal gain profile and intensity distribution are given by (18) and (19), respectively. For the constant and quadratic term of the amplitude gain we find

$$
\begin{aligned}
& \alpha_{0}=\frac{\frac{1}{2} G}{\left\{1+I_{t} / I_{0}\right\}^{1 / 2}} \\
& \alpha_{2}=\frac{\frac{1}{2} G}{\left\{1+I_{t} / I_{0}\right\}^{1 / 2}}\left(\gamma+\frac{2}{\omega^{2}} \frac{I_{t} / I_{0}}{1+I_{t} / I_{0}}\right) .
\end{aligned}
$$

By substituting (27) and (28) into (14), the incremental gain of an inhomogeneous transition becomes

$$
\frac{d \ln \left(I \omega^{2}\right)}{d z}=\frac{G}{\left\{1+I_{t} / I_{0}\right\}^{1 / 2}}\left(1+\frac{1}{4} \gamma \omega^{2}+\frac{1}{2} \frac{I_{t} / I_{0}}{1+I_{t} / I_{0}}\right)
$$

If the small-signal gain profile of gas lasers is again proportional to the electron density and therefore approximated by a zero-order Bessel function, we find, similar to the homogeenous case, that the threshold condition to start oscillation requires much higher inversion densities along the axis than by ignoring this gain profile. With $\omega^{2} / \rho^{2}$ about 0.5 , the threshold inversion density is about 50 percent higher. Further, we see that for $I_{t} / I_{0} \gg 1$ the contribution of radial radiation transport to the incremental gain due to the Gaussian intensity distribution is about equal to half the amount of stimulated emission given by the first term on the right-hand side of (29).

In order to find the intensity characteristics, we integrate (29) over a round trip through the resonator and consider again the case where the beamwidth varies little within the resonator, so that it can be approximated as constant. We find, similar to the homogeneous case,

$$
-\frac{\ln R}{2 G L}=\frac{1}{\left\{1+I_{t} / I_{0}\right\}^{1 / 2}}\left(1+0.25 \gamma \omega_{+}^{2} \frac{0.5 I_{t} / I_{0}}{1+I_{t} / I_{0}}\right) .
$$

The normalized output intensities are plotted in Figs. 3 and 4. It is seen that the obtained characteristics also predict a start jump as one slowly increases the gain over loss ratio and passes threshold. Again, the jump only exists for negative values of $\gamma$ and increases with decreasing $\gamma$. By comparing this result with the numerical evaluation of gain saturation as given by Fox and $\mathrm{Li}$ [8], it is seen that they did not find a start jump. The reason may be that they consider a homogeneous small-signal gain coefficient $(\gamma=0)$, which according to our results will indeed not lead to a start jump.

\section{Start Jump of a Gaussian Mode}

The aforementioned predicted start effect can be demonstrated experimentally. It can be found either by increasing the pumping power or by decreasing the outcoupling losses from below threshold. For continuously variable outcoupling losses we chose a Gaussian-mode $\mathrm{CO}_{2}$ laser with an outcoupling cavity. The experimental setup is indicated in Fig. 5. The cavity has a length of $150 \mathrm{~cm}$ and an internal diameter of $9 \mathrm{~mm}$. There is a totally reflecting gold mirror with a radius 


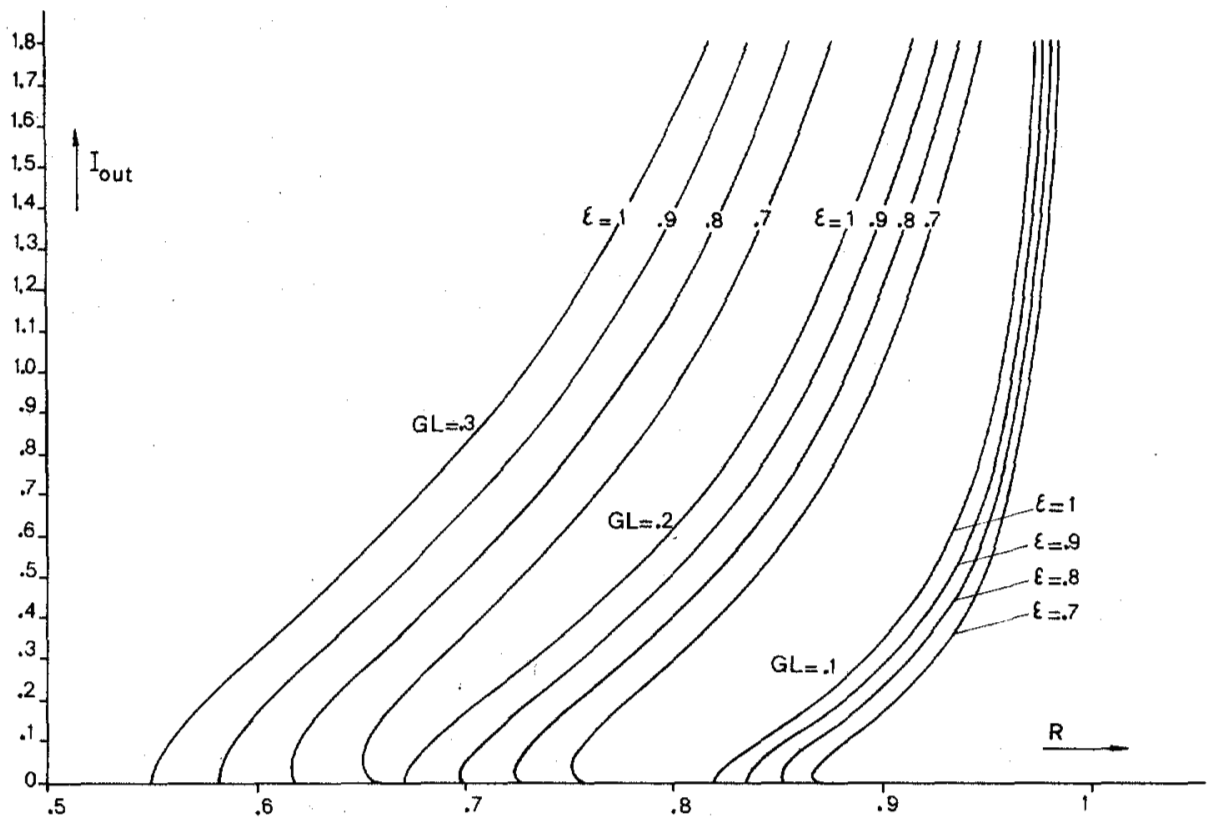

Fig. 4. The outcoupled intensity for inhomogeneous transitions is plotted versus reflectivity for various values of the small-signal gain and its profile parameter $\epsilon$.

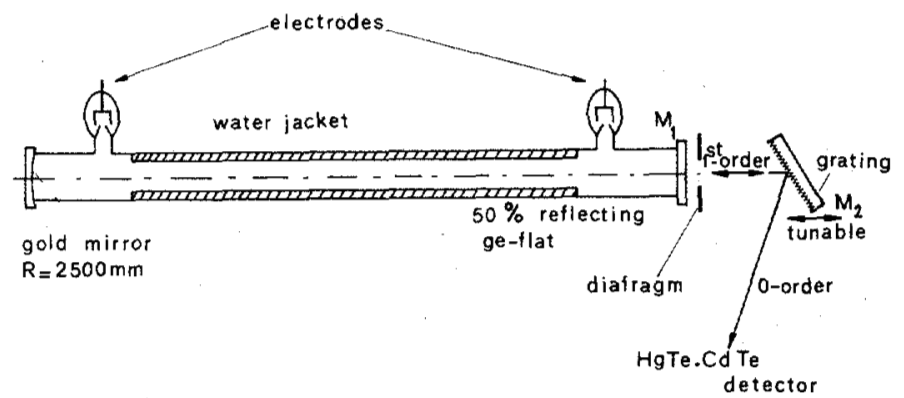

Fig. 5. Experimental arrangement of a Gaussian-mode laser with transition selective outcoupling cavity. The outcoupling is continuously variable. The grating is mounted on a tunable piezoelectric translator.

of curvature of $2500 \mathrm{~mm}$ on one side and a 50-percent reflecting germanium flat on the other side. The length of the discharge is about $130 \mathrm{~cm}$. With a mixture of about 8 torr $\mathrm{CO}_{2}$ and 6 torr $\mathrm{He}$, the cavity is overcoupled and will not lase with a dc discharge current of about $10 \mathrm{~mA}$. By placing a wavelength selective grating in front of the germanium flat, we use the first-order reflection on the grating for intracavity reflection and the zero-order reflection for outcoupling. The grating, mounted on a tunable piezoelectric translator for position scanning, together with the germanium flat, forms a Fabry-Perot (FP). This FP as an outcoupling cavity offers a variable narrow-band reflection [7]. In order to suppress higher order modes, a $4.2 \mathrm{~mm}$ diaphragm is placed between the grating and the germanium flat. The diffraction grating is gold coated, has 75 lines $/ \mathrm{mm}$, and is blazed for $10.6 \mu$. The outcoupled radiation is detected with a fast $\mathrm{HgTe}: \mathrm{CdTe}$ photo diode having a time constant of a few nanoseconds.

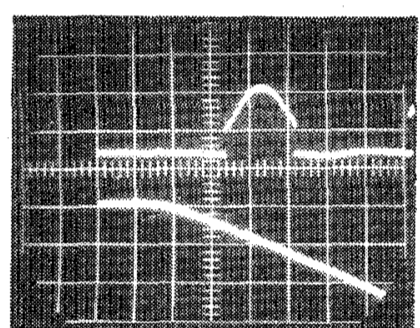

Fig. 6. The outcoupled intensity as a function of the position scanning of the piezoelectric translator. The reflectivity of the coupling cavity first increases and further on decreases with the translated position of the grating. The lower part shows the ramp voltage. The time scale is $5 \mathrm{~ms} / \mathrm{cm}$.

Fig. 6 shows the output intensity as a function of the position scanning of the piezoelectric translator. The lower part shows the ramp voltage applied to the translator and the upper part shows the output as a function of time with $5 \mathrm{~ms} /$ division. The reflectivity is continuously varied by the applied ramp voltage from below threshold. At threshold the intensity does not increase continuously but clearly jumps. It also falls suddenly back to zero as the reflectivity is decreased by further scanning.

By using the laser system without the grating, varying gain is obtained by increasing the current suddenly from 1 to $40 \mathrm{~mA}$. (In a dc experiment it will not lase at $40 \mathrm{~mA}$ ) After this change the inversion density grows and passes threshold and after some time the inversion drops again, probably due to thermal effects. The upper trace of Fig. 7 shows the change of current and the lower trace shows the radiation output. The time scale is $50 \mu \mathrm{s} /$ division. Apparently, it takes some 


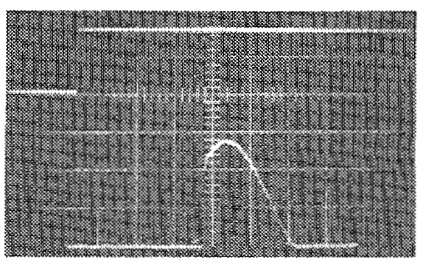

Fig. 7. The de discharge current of about $1 \mathrm{~mA}$ is suddenly changed to $40 \mathrm{~mA}$, as shown by the upper trace. The output of the laser without grating and diaphragm is shown by the lower trace. The time scale is $50 \mu \mathrm{s} / \mathrm{cm}$.

time before the threshold inversion is built up, and then the oscillation jumps to a certain intensity. The extinction of the laser action is not sudden. It may be that, for the steady state of $40 \mathrm{~mA}$ due to thermal effects, the small-signal gain profile becomes flat or even reversed. If the same experiment is done by suddenly increasing the current from about $5 \mathrm{~mA}$, where the steady-state oscillations exist, to $40 \mathrm{~mA}$, it is found that the oscillating field increases continuously and finally drops to zero. This is shown in Fig. 8, where the time scale is $100 \mu \mathrm{s} /$ division.

\section{Conclusions}

A three-dimensional treatment of the interaction of a Gaussian mode and a saturated medium leads to conclusions that differ much from one-dimensional treatment. We wish to stress again that the gain parameters have practically no influence on the intensity distribution, i.e., there is no focusing owing to the gain parameters, but they have a large influence on the energy flux and output power. For both homogeneous and inhomogeneous transitions, the effect of the Gaussian distribution of the intensity on the saturation of the medium is a considerable increase of output power. Finally, it is found

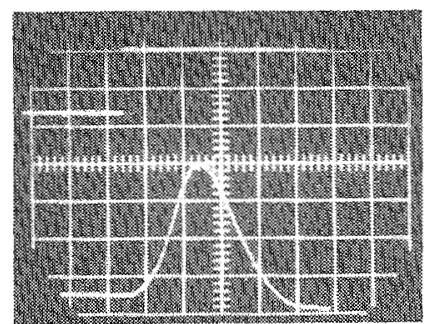

Fig. 8. A dc discharge of about $5 \mathrm{~mA}$ producing a weak laser field is suddenly changed to $40 \mathrm{~mA}$, as shown by the upper trace. The Gaussian radiation intensity is shown to be continuous, and it finally goes to zero. The time scale is $100 \mu \mathrm{s} / \mathrm{cm}$.

that near threshold the intensity is not continuously variable but jumps.

\section{REFERENCES}

[1] W. W. Rigrod, "Saturation effects in high-gain lasers," J. Appl. Phys., vol. 36, pp. 2487-2490, Aug. 1965.

[2] P. W. Smith, "The output power of a $6328-\AA$ He-Ne gas laser," IEEE J. Quantum Electron., vol. QE-2, pp. 62-68, Mar. 1966.

[3] G. J. Ernst and W. J. Witteman, "Mode structure of active resonators," IEEE J. Quantum Electron., vol. QE-9, pp. 911-918, Sept. 1973.

[4] H. Kogelnik, "On the propagation of Gaussian beams of light through lenslike media including those with loss or gain variation," Appl. Opt., vol. 4, pp. 1562-1569, Dec. 1965.

[5] W. W. Rigrod, "Gain saturation and output power of optical masers," J. Appl. Phys., vol. 34,pp. 2602-2609, Sept. 1963.

[6] G. J. Ernst and W. J. Witteman, "The effect of radial radiation transport on intensity characteristics and oscillation frequency of homogeneously broadened lasers," IEEE J. Quantum Electron., vol. QE-10, pp. 37-44, Jan. 1974.

[7] _- "Transition selection with adjustable outcoupling for a laser device applied to $\mathrm{CO}_{2}$," IEEE J. Quantum Electron., vol. QE-7, pp. 484-488, Oct. 1971.

[8] A. G. Fox and T. Li, "Effect of gain saturation on the oscillating modes of optical masers," IEEE J. Quantum Electron,, vol. QE-2, pp. 774-783, Dec. 1966. 